\title{
TWO NEW POINTS IN THE SPECTRUM OF THE ABSOLUTE MAHLER MEASURE OF TOTALLY POSITIVE ALGEBRAIC INTEGERS
}

\author{
V. FLAMMANG
}

\begin{abstract}
For totally positive algebraic integers $\alpha \neq 0,1$ of degree $d(\alpha)$, we consider the set $\mathcal{L}$ of values of $M(\alpha)^{\frac{1}{d(\alpha)}}=\Omega(\alpha)$, where $M(\alpha)$ is the Mahler measure of $\alpha$. C. J. Smyth has found the four smallest values of $\mathcal{L}$ and conjectured that the fifth point is $\Omega\left(\left(2 \cos \frac{2 \pi}{60}\right)^{2}\right)$. We prove that this is so and, moreover, we give the sixth point of $\mathcal{L}$.
\end{abstract}

\section{INTRODUCTION}

Let $P=a_{0} x^{d}+\cdots+a_{d}=a_{0}\left(x-\alpha_{1}\right) \cdots\left(x-\alpha_{d}\right), a_{0} a_{d} \neq 0$, be a polynomial of degree $d \geq 1$ with complex coefficients.

The relative Mahler measure of $P$ is given by $M(P)=\left|a_{0}\right| \prod_{i=1}^{d} \max \left(1,\left|\alpha_{i}\right|\right)$.

We denote by $\Omega(P)=M(P)^{\frac{1}{\operatorname{deg}(P)}}$ the absolute Mahler measure of $P$ and by $\mathcal{L}$ the set of $\Omega(P)$ where $P$ is a monic polynomial with integer coefficients, different from $x, x-1$, irreducible over $\mathbb{Q}$ and totally positive (i.e., all its roots are real and positive). The absolute Mahler measure of a nonzero algebraic integer $\alpha$ is the absolute Mahler measure of its minimal polynomial in $\mathbb{Z}[x]$.

We define $\alpha_{n}=2 \cos (2 \pi / n)$ and the real numbers $\left(\beta_{n}\right)_{n \geq 0}$ by

$$
\left\{\begin{array}{l}
\beta_{0}=1 \\
\beta_{n+1}>0 \text { and } H \beta_{n+1}=\beta_{n} \text { for } n \geq 0
\end{array}\right.
$$

where

$$
H x=x-\frac{1}{x} .
$$

In 1981, C.J. Smyth [4] found the four smallest elements of $\mathcal{L}$, which are

$$
\begin{aligned}
& \Omega\left(\beta_{1}^{2}\right) \approx 1.618033, \quad \Omega\left(\beta_{2}^{2}\right) \approx 1.685389 \\
& \Omega\left(\beta_{3}^{2}\right) \approx 1.710197, \quad \Omega\left(\alpha_{7}^{2}\right) \approx 1.715534 .
\end{aligned}
$$

He had conjectured that the fifth point of $\mathcal{L}$ was $\Omega\left(\alpha_{60}^{2}\right) \approx 1.719388$.

In this paper, we show

Received by the editor February 1, 1994.

1991 Mathematics Subject Classification. Primary 11R06, 11J68. 
Theorem. Each monic polynomial $P$ of degree d, with integer coefficients and totally positive satisfies

$$
\Omega(P) \geq 1.720566 \cdot \prod_{j=0}^{9}\left|\operatorname{resultant}\left(P, R_{j}\right)\right|^{\frac{c_{j}}{d}} .
$$

In particular, if $P$ is not divisible by any of the $R_{j}, 0 \leq j \leq 9$, then

$$
\Omega(P) \geq 1.720566
$$

Here,

$R_{0}(x)=x-1$,

$R_{1}(x)=x^{2}-3 x+1$,

$R_{2}(x)=x^{4}-7 x^{3}+13 x^{2}-7 x+1$,

$R_{3}(x)=x^{8}-15 x^{7}+83 x^{6}-220 x^{5}+303 x^{4}-220 x^{3}+83 x^{2}-15 x+1$,

$R_{4}(x)=x^{16}-31 x^{15}+413 x^{14}-3141 x^{13}+15261 x^{12}-50187 x^{11}+115410 x^{10}$

$-189036 x^{9}+222621 x^{8}-189036 x^{7}+115410 x^{6}-50187 x^{5}+15261 x^{4}-3141 x^{3}$

$+413 x^{2}-31 x+1$,

$R_{5}(x)=x^{4}-8 x^{3}+15 x^{2}-8 x+1$,

$R_{6}(x)=x$,

$R_{7}=\left(x^{3}-5 x^{2}+6 x-1\right) \cdot\left(x^{3}-6 x^{2}+5 x-1\right)$,

$R_{8}=\left(x^{4}-7 x^{3}+14 x^{2}-8 x+1\right) \cdot\left(x^{4}-8 x^{3}+14 x^{2}-7 x+1\right)$,

$R_{9}=x^{6}-12 x^{5}+44 x^{4}-67 x^{3}+44 x^{2}-12 x+1$

and

$$
\begin{array}{ll}
c_{0}=0.32506177, & c_{1}=0.04536243, \\
c_{2}=0.01688943, & c_{3}=0.00569441, \\
c_{4}=0.00195846, & c_{5}=0.00118560, \\
c_{6}=0.20333607, & c_{7}=0.00292360, \\
c_{8}=0.00069160, & c_{9}=0.00087933 .
\end{array}
$$

Note that $\alpha_{7}^{2}$ and $\alpha_{7}^{-2}$ are roots of $R_{7}$ and $\alpha_{60}^{2}$ and $\alpha_{60}^{-2}$ are roots of $R_{8}$. Note also that $H^{3}\left(\alpha_{7}\right)=-\alpha_{7}$ and $H^{4}\left(\alpha_{60}\right)=\alpha_{60}$.

Corollary. The first six elements of $\mathcal{L}$ are

$$
\Omega\left(\beta_{1}^{2}\right), \Omega\left(\beta_{2}^{2}\right), \Omega\left(\beta_{3}^{2}\right), \Omega\left(\alpha_{7}^{2}\right), \Omega\left(\alpha_{60}^{2}\right), \Omega\left(\beta_{4}^{2}\right) .
$$

These are the only points of $\mathcal{L}$ in the interval $(1,1.720556]$.

Remark. C.J. Smyth [3] showed that $\mathcal{L}$ is everywhere dense in $(l, \infty)$, where $l \approx$ 1.7273 is the first accumulation point known.

The proof follows Smyth's [4]. In the second paragraph, we recall briefly the methods. In paragraph 3 , we will describe the arguments which permit us to get a general result on $\Omega(P)$ improving Smyth's theorem.

\section{Principle of AUXiliary Functions}

For $x>0$, put $\log _{+}(x)=\max (0, \log (x))$. For $n \geq 1$, let $c=\left(c_{j}\right)_{1 \leq j \leq n}$ be nonnegative real numbers and $R_{j}, 0 \leq j \leq n$, polynomials in $\mathbb{Z}[x]$. 
For $x>0$, we consider the auxiliary function

$$
f(x, c)=\log _{+}(x)-\sum_{j=0}^{n} c_{j} \log \left|R_{j}(x)\right|
$$

and $m(c)=\min _{x>0} f(x, c)$.

The polynomial $P$ with integer coefficients, which is irreducible over $\mathbb{Q}$, has positive zeros $\alpha_{1}, \ldots, \alpha_{d}$ and is not dividing any of the $R_{j}$, satisfies

$$
\left|\operatorname{resultant}\left(P, R_{j}\right)\right|=\prod_{i=1}^{d}\left|R_{j}\left(\alpha_{i}\right)\right| \geq 1, \quad j=0, \ldots, n,
$$

which implies

$$
\log _{+}\left(\alpha_{i}\right) \geq m(c), \quad i=1, \ldots, d,
$$

and therefore,

$$
\Omega(P) \geq e^{m(c)} .
$$

We then have to solve the following optimization problem: determine

$$
m=\max _{c} m(c)=\max _{\left(c_{j}\right)} \min _{x>0} f(x, c) .
$$

A semi-infinite linear programming method gives good numerical values for the $c_{j}$. For more details, one can refer to [4] or [2].

\section{NUMERICAL RESUltS}

The proof of the theorem is based on four main points : first, a better choice of polynomials in the auxiliary function, and then three numerical improvements in the semi-infinite linear programming methods.

3.1. The auxiliary function. C.J. Smyth [3] has used a transformation $T$ which is essential in his paper : to a totally positive polynomial $P$ of degree $d$, one associates the reciprocal polynomial $T(P)$ (i.e., $\left.T(P)(x)=x^{\operatorname{deg}(T(P))} \cdot T(P)\left(\frac{1}{x}\right)\right)$ of degree $2 d$ and also totally positive defined by

$$
T(P)(x)=x^{d} \cdot P\left(x+\frac{1}{x}-2\right) .
$$

We define the $n$th descendant of a polynomial $P$ the polynomial $T^{n}(P)$ defined by

$$
T^{n}(P)(x)=x^{\operatorname{deg}\left(T^{n-1}(P)\right)} \cdot T^{n-1}(P)\left(x+\frac{1}{x}-2\right) .
$$

The minimal polynomial $P_{n}$ of $\beta_{n}^{2}$ is the $n$th descendant of $P_{0}(x)=x-1$.

The $R_{j}, j=0, \ldots, 5$, belong to two particular families of polynomials. The first consists of iterates by $T$ of $P_{0}=x-1$. Smyth [4] already used $R_{j}=P_{j}=T^{j}\left(P_{0}\right)$, $j=0, \ldots, 3$. We add $R_{4}=P_{4}$, of degree 16 . The second family appears here for the first time. It is composed of iterates by $T$ of $Q_{0}=x-2$. We need

$$
R_{5}=T^{2}\left(Q_{0}\right)=x^{4}-8 x^{3}+15 x^{2}-8 x+1 .
$$


Although this new family occurs only with one of its polynomials, this one is absolutely necessary to get the result.

Remark. Let $G=H^{2}$ be defined for $x>0$ by

$$
G x=x+\frac{1}{x}-2 .
$$

The fixed point of $G$ is the root of the polynomial $Q_{0}^{\prime}=1-2 x$ whose reciprocal polynomial is $Q_{0}$.

Note that $R_{9}$ is used here for the first time and is the first descendant of the polynomial $x^{3}-6 x^{2}+5 x-1$.

For $x>0$ such that $R_{j}(x) \neq 0,0 \leq j \leq 9$, the function $f(x, c)$ becomes

$$
f(x, c)=\log _{+}(x)-\sum_{j=0}^{9} c_{j} \log \left|R_{j}(x)\right| .
$$

As it is composed of $x, x-1$ and reciprocal polynomials, we can use the remark of $[2, \S 2]$ to impose $f(x)=f(1 / x)$ and limit the study of $f$ to the interval $(0,1)$.

This condition implies that:

$$
2 c_{6}+\sum_{j=0, j \neq 6}^{9} c_{j} \cdot \operatorname{deg}\left(R_{j}\right)=1 .
$$

3.2. Numerical improvements. Semi-infinite linear programming is an iterative method. At each step, we have to :

(a) determine $\min _{x>0} f(x, c)$ for a particular family of coefficients $c=\left(c_{j}\right)$;

(b) increase the set of points on which the function is controlled;

(c) evaluate $\log \left|P_{j}\left(x_{i}\right)\right|$ at control points $x_{i}$.

For (a), we use a computational algorithm (AMOEBA [5]) giving the minimum of a function without calculating its derivative. This technique reduces the risk of computational errors that can occur when the numerator of the derivative has high degree ( here, 55).

For (b), there are two common procedures: either use a method similar to the Remes algorithm [1], or add systematically all points where the derivative $f^{\prime}$ of $f$ vanishes. We choose an intermediate method consisting in adding points where not only $f^{\prime}=0$ but also $f$ is small. This avoids saturation and guarantees fast convergence. For example, at the $i$ th iteration, for a particular family of coefficients $c$, we decide to remove points $y>0$ not satisfying

$$
f(y, c)<\min _{x>0} f(x, c)+\epsilon
$$

for suitable $\epsilon>0$.

(c) Let $x_{0}$ be a control point of $f$. We consider the polynomials $P_{1}, P_{2}, P_{3}, P_{4}$ derived from $P_{0}$. Instead of evaluating directly $\log \left|P_{j}\left(x_{0}\right)\right|, j=1, \ldots, 4$, we take advantage of the definition of these $P_{j}$. Remember that $P_{j}=T^{j}\left(P_{0}\right)$. By recurrence, we can easily show that

$$
\log \left|P_{j}\left(x_{0}\right)\right|=\sum_{k=1}^{j} \log \left|x_{j-k}\right|+\log \left|x_{j}-1\right|
$$


with

$$
x_{k+1}=x_{k}+\frac{1}{x_{k}}-2 .
$$

This procedure is easy to use and permits to limit the computational errors when the degree of polynomials becomes high.

Using semi-infinite linear programming, after 15 iterations we obtain

$$
1.72056628 \leq e^{m} \leq 1.7205664
$$

But the accuracy of the calculations is not guaranteed, especially for the zeros of $f^{\prime}$ whose numerator has degree 55. They have been checked by the formal computational system PARI with great precision and we so find the constant 1.720566 announced in the theorem.

\section{Conclusion}

All the above results seem to show that the elements of $\mathcal{L}$ in the interval $\left(\frac{1+\sqrt{5}}{2}, l\right)$ are only of two forms : absolute measures of $\beta_{n}^{2}$ or, as suggests C.J.Smyth, of fixed points of iterates of the transformation $T$. But we believe that this conjecture cannot be resolved with the methods used in this paper.

\section{ACKNOWLEDGMENTS}

I thank Georges Rhin for his collaboration in the improvements of the numerical methods used in this paper and Michel Olivier for his help in the computations with PARI.

\section{REFERENCES}

1. E.W. Cheney, Introduction to approximation theory, McGraw-Hill, New York, 1966. MR 36:5568

2. V. Flammang, Sur la longueur des entiers algébriques totalement positifs, J. Number Theory (to appear).

3. C.J. Smyth, On the measure of totally real algebraic integers. I, J. Austral. Math. Soc. (Ser. A) 30 (1980), 137-149. MR 82j:12002a

4. _ On the measure of totally real algebraic integers. II, Math. Comp. 37 (1981), 205-208. MR 82j: $12002 b$

5. W.H. Press, B.P. Flannery, S.A. Teukolsky, and W.T. Vetterling, Numerical recipes, The Art of Scientific Computing, Cambridge Univ. Press, Cambridge, 1986. MR 87m:65001a

URA CNRS No 399, Département de Mathématiques et Informatique, U.F.R. Mim. Université de Metz, Ile du Saulcy, 57045 Metz, Cedex 1, France

E-mail address: flammang@poncelet.univ-metz.fr 